\title{
Prostatic Artery Embolization through the Profunda Femoris Artery
}

\author{
Abin Sajan ${ }^{1, \odot ~ A r i ~ I s a a c s o n ~}{ }^{2}$ Sandeep Bagla ${ }^{3}$ \\ ${ }^{1}$ Department of Surgery, NYU Winthrop Hospital, Mineola, New \\ York, United States \\ ${ }^{2}$ Department of Radiology, University of North Carolina at Chapel \\ Hill School of Medicine, Chapel Hill, North Carolina, United States \\ ${ }^{3}$ Department of Interventional Radiology, Vascular Interventional \\ Partners - NOVA, Falls Church, Virginia, United States
}

\begin{abstract}
Address for correspondence Ari Isaacson, MD, Department of Radiology, University of North Carolina at Chapel Hill School of Medicine, 101 Manning Drive, Chapel Hill, NC 27514, United States (e-mail: Ari_isaacson@med.unc.edu).
\end{abstract}

J Clin Interv Radiol ISVIR:2021;5:48-50

\begin{abstract}
An 88-year-old catheter-dependent patient with peripheral vascular disease and benign prostatic hyperplasia was referred for prostatic artery embolization (PAE). Selective angiography of the left internal iliac artery (IIA) revealed a $2.5-\mathrm{cm}$ aneurysm and a single artery comprising the anterior division of the IIA (inferior gluteal artery). No prostatic artery (PA) was identified on the left. Additional angiography of the left external iliac artery (EIA), right IIA, and right EIA did not demonstrate prostatic perfusion. The right profunda femoris artery was then selected given previously reported atherosclerotic collaterals, which revealed an ascending branch of the medial circumflex femoral artery coursing through the upper thigh into the pelvis and reconstituting the right anterior division of the IIA. A 2.4-French microcatheter (Terumo)

Keywords

- prostate artery

- embolization

- benign prostatic hyperplasia and 0.018 -inch wire (Transcend, Boston Scientific) were used to enter the pelvic vasculature, right PA was identified, and a 2-mm coil was used to perform embolization of a potential nontarget anterior division branch. Embolization of the right PA was then performed with $250-\mu \mathrm{m}$ particulate and Gelfoam slurry. Two-week follow-up revealed spontaneous voiding and catheter independence.
\end{abstract}

\section{Introduction}

Prostatic artery embolization (PAE) is a challenging procedure due to the complexity of anatomical variations of the prostatic artery. The prostatic artery can arise from any branch of the internal iliac artery (IIA), but most commonly it arises from a branch of the anterior division. On rare occasions, it can even originate from the external iliac artery. ${ }^{1}$ In addition, when the prostatic arteries are occluded, either from atherosclerotic disease or prior embolization, new collateral pathways can form, adding more variability to the prostatic arterial supply. ${ }^{2}$ What follows is a case describing a unique prostatic artery collateral in a patient with atherosclerotic plaque occluding the native prostatic artery. Retrospective case reports are exempt from review by the Institutional Review Board at our institution.

\section{Case Presentation}

An 88-year-old catheter-dependent patient with a history of coronary artery disease and benign prostatic hyperplasia was referred for PAE. He had a history of coronary artery disease treated with quadruple bypass as well as peripheral arterial disease of the lower extremities previously treated with stent placement.

Preprocedural magnetic resonance imaging (MRI) revealed a prostate volume of $220 \mathrm{~cm}^{3}$. International published online January 25, 2021
Dol https://doi.org/

10.1055/s-0041-1723060

ISSN 2457-0214. (c) 2021. Indian Society of Vascular and Interventional Radiology. This is an open access article published by Thieme under the terms of the Creative Commons Attribution-NonDerivative-NonCommercial-License, permitting copying and reproduction so long as the original work is given appropriate credit. Contents may not be used for commercial purposes, or adapted, remixed, transformed or built upon. (https://creativecommons.org/licenses/by-nc-nd/4.0/).

Thieme Medical and Scientific Publishers Pvt. Ltd. A-12, 2nd Floor, Sector 2, Noida-201301 UP, India 
Prostate Symptom Score was not measured since the patient was catheter-dependent. Selective angiography of the left IIA through right femoral artery access revealed a $2.5-\mathrm{cm}$ aneurysm and a single artery comprising the anterior division of the IIA (inferior gluteal artery). No prostatic artery was identified ( $\mathbf{- F i g . ~ 1 B )}$. Angiography of the left external iliac artery also did not demonstrate perfusion of the prostate. The right IIA was then selected, and angiography demonstrated anterior division occlusion ( - Fig. 1A). Additional angiography of the right external iliac artery was also noncontributory. The right profunda femoris artery (PFA) was then selectively catheterized using a reverse curve SOS2 catheter (AngioDynamics) and a 2.4-French microcatheter (Terumo Medical Corp.), which revealed a small ascending branch of the medial circumflex femoral artery coursing through the upper thigh into the pelvis and reconstituting the right anterior division of the IIA (-Fig. 2).

A 2.4-French microcatheter (Terumo) and 0.018-inch wire (Transcend, Boston Scientific) were used to advance through the torturous collateral branch to enter the pelvic vasculature. The right prostatic artery was then identified, and a 2-mm coil was used to perform embolization of a potential nontarget anterior division branch. Following administration of $200 \mathrm{mcg}$ of Nitroglycerin (American Regent), embolization of the PA was performed with $250-\mu \mathrm{m}$ Embozene particulates and Gelfoam slurry (-Fig. 3). Embolization was then performed from this artery. Two weeks later, the patient was able to void spontaneously and no longer required a bladder catheter.

\section{Discussion}

The case in this study describes embolization of the prostate from an artery with a unique origin arising from the PFA. This scenario underscores the potential for prostatic blood supply to collateralize through various pathways in the setting of atherosclerotic occlusion of the native prostatic artery. This phenomenon adds to the technical difficulty and anatomical variability of an already challenging procedure.
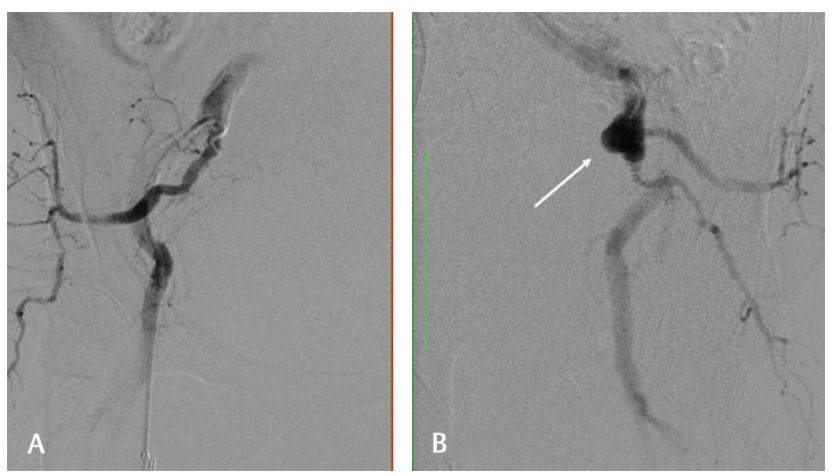

Fig. 1 (A) Right anterior oblique digital subtraction angiography (DSA) of the right IIA demonstrating occlusion of the anterior division and no apparent prostatic artery. (B) Left anterior oblique DSA of the left IIA demonstrating a $2.5-\mathrm{cm}$ aneurysm (arrow) and no apparent prostatic artery.
Although not acquired prior to this PAE, preprocedural computed tomography angiography (CTA) or magnetic resonance angiography (MRA) can be helpful to determine the origin of the prostatic arterial supply prior to starting the procedure. Intraprocedural cone beam CTA can also be useful when the prostatic artery is not identified on angiography. Cone beam CTA was not used in the present case given the operator's extensive experience with performing PAE. Cone beam CTA can be performed from the aorta or IIA reliably allowing for identification of the prostatic artery origins.

We suspected a lower extremity collateral given the patient's history of long-standing occlusive arterial disease. Our suspicion was driven by previous experience in the treatment of complex pelvic aneurysm endoleaks, where supply to the hypogastric branches could be catheterized from PFA branches. It has also been previously reported in the surgical literature that prominent iliofemoral collaterals develop in the setting of chronic iliofemoral occlusive disease. ${ }^{3}$ This case describes a rare origin of the PA and highlights the importance of considering patient history when unexpected clinical findings arise.
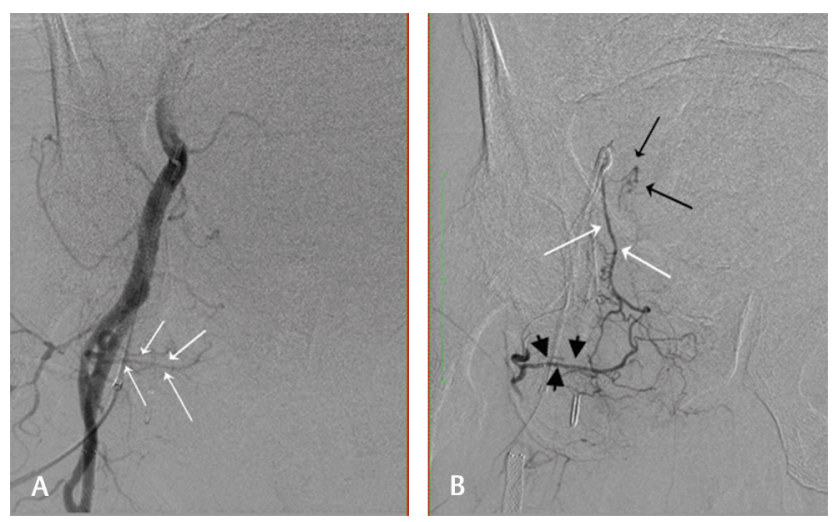

Fig. 2 (A) Right anterior oblique digital subtraction angiography (DSA) of right external iliac artery demonstrating a medial circumflex femoral artery (MCFA) (arrows) arising from the profunda femoris artery and coursing toward the medial pelvis. (B) Right anterior oblique DSA after selective catheterization of the MCFA. The obturator artery (white arrows) is reconstituted by the MCFA (arrowhead) and demonstrates a right prostatic artery (black arrows).
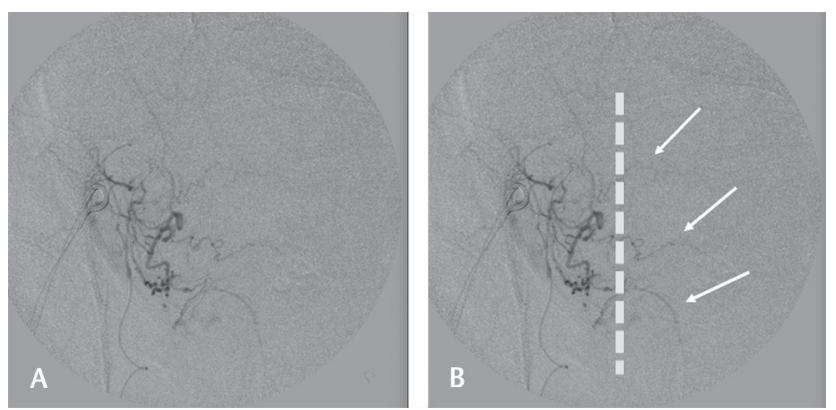

Fig. 3 (A) Right anterior oblique DSA after selective catheterization of the right prostatic artery through the profunda femoris artery pathway. (B) The dashed line separates the left and right hemiprostate. Multiple branches of the right hemiprostate are seen with branches (white arrows) perfusing the left hemiprostate. 
50 Prostatic Artery Embolization through the Profunda Femoral Artery Sajan et al.

\section{Disclosure}

Dr. Bagla is a consultant for Boston Scientific, Embolics, Terumo, Medtronic, and Teleflex and reports other fees from Boston Scientific, Varian Medical Systems, Medtronic, Embolx, IMBiotechnologies, and Phillips Medical System, outside the submitted work. Dr. Isaccson is a consultant for Boston Scientific, Embolics, Terumo, and BTG and reports other fees from Terumo, ABK Biomedical, and CrannMed, outside the submitted work.

\section{Conflict of Interest}

None declared.

\section{References}

1 Carnevale FC, Soares GR, de Assis AM, Moreira AM, Harward SH, Cerri GG. Anatomical variants in prostate artery embolization:apictorialessay.CardiovascInterventRadiol2017; 40(9):1321-1337

2 Bagla S, Smirniotopolous JB, Vadlamudi V. Crossing a prostatic artery chronic total occlusion to perform prostatic arterial embolization. J Vasc Interv Radiol 2016;27(2):295-297

3 Hashemi H, Massimiano P, Mukherjee D, Aryavand B. Internal iliac artery revascularization for limb salvage purposes. J Vasc Surg 2007;45(3):607-609 